\title{
Use of Methods of Wave Processes Modeling for Control of Defectiveness of Non-Metallic Materials
}

\author{
T. V. Fursa, ${ }^{1, \text { a) }}$, B. A. Lyukshinn, b) , G. E. Utsyn ${ }^{1, \text { a) }}$, and M. V. Kuimova ${ }^{1, c)}$ \\ ${ }^{1}$ National Research Tomsk Polytechnic University, Tomsk, 634050, Russia \\ ${ }^{2}$ Institute of Strength Physics and Materials Science SB RAS, Tomsk, 634055, Russia \\ a) Corresponding author: uge23@rambler.ru \\ b)1ba2008@yandex.ru \\ c)mkuimova@mail.ru
}

\begin{abstract}
The paper presents theoretical and experimental research of an electrical response to the elastic impulse excitation of concrete samples. The authors describe three-dimensional modeling and 3D visualization of wave processes and calculate the parameters of an electrical signal from defect-free and defective samples. The research demonstrates the comparison of design data with experimental outcomes; good precision of data was obtained.
\end{abstract}

Keywords: mechanoelectric transformations, electrical response, 3D modelling, wave processes, defect, non-metallic materials

\section{INTRODUCTION}

The problem of flaw detection in engineering constructions made of heterogeneous non-metallic materials (e.g. concrete) with high reliability requirements is of high importance. Surface cracks are the most widespread type of construction defects. The reasons of cracks occurrence and development are static and dynamic loads, deterioration processes, drying shrinkage, climate effects and other breakdown processes.

Methods that help to detect and characterize faults by means of non-destructive testing are of interest for civil engineers [1]. Engineers widely use ultrasonic and acoustic methods of non-destructive testing [2] and apply surface waves methods [3]. In the recent years, extensive research in the development of non-contact non-destructive testing methods of concrete has been conducted. The research outcomes of non-contact reading of surface waves characteristics in concrete using laser are provided in research works [4, 5]. Built-in piezoids are also suggested for use for a non-contact method of acoustic vibrations registration [6]. The existing methods are characterized by poor accuracy and low sensitivity to internal defects and non-homogeneity. This problem can be solved with the use of mechanoelectric transformations in heterogeneous dielectric materials at pulse elastic excitement [7, 8]. The essence of this phenomenon is that the impact of acoustic waves on the sources of dynamoelectric transformations generates an alternating electric field. The acoustic waves are formed in the sample of a finite size at its shock excitement. The prospective of using electrical responses parameters to elastic excitement for defect detection in concrete was confirmed by the results of the previous research [9].

\section{THEORETICAL AND EXPERIMENTAL STUDIES}

The configuration of the impact system, the source inside the sample and the set point of the receiver are shown in Fig. 1. The bias current equation is:

$$
I_{s m}(t)=K \int_{S_{d}} \frac{V(t)}{r^{3}}\left(\frac{3 h^{2}}{r^{2}}-1\right) \mathrm{d} S_{d},
$$

International Conference on Physical Mesomechanics of Multilevel Systems 2014 AIP Conf. Proc. 1623, 163-166 (2014); doi: 10.1063/1.4898908

(C) 2014 AIP Publishing LLC 978-0-7354-1260-6/\$30.00 


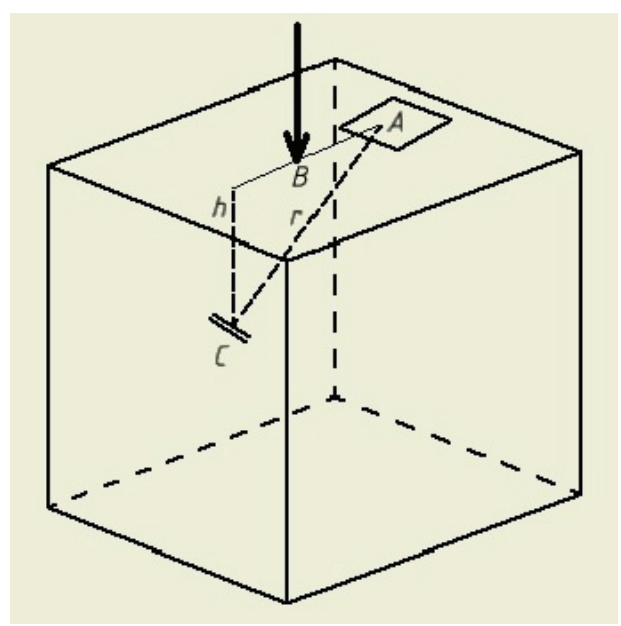

FIGURE 1. Schematic arrangement of a piezoelectric source in the sample relating to the set element of the electrical receiver

where $K$-the constant depending on the module of the elasticity of the material, the amount of piezoelectric inclusion and the sample sizes; $V(t)$ - the drift velocity; $h$ - the depth of piezoelectric source arrangement; $r$ - the distance from the source to the receiver electrode; $S_{d}$ - the area of the measuring electrode. A detailed calculation of physico-mathematical model of electrical response is presented in the following paper [10]. The numerical algorithm is based on the Runge-Kutta method for solving ordinary differential equations. This approach results from the second order non-central scheme offered by MacCormack [11].

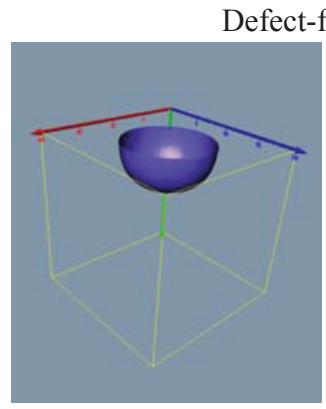

$[7 \mu \mathrm{s}]$

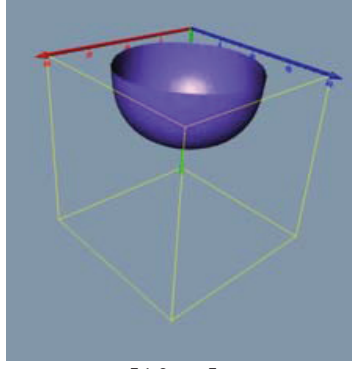

$[10 \mu \mathrm{s}]$

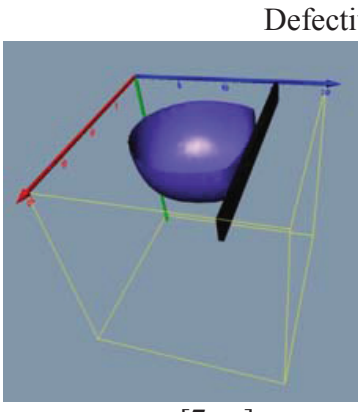

$[7 \mu \mathrm{s}]$

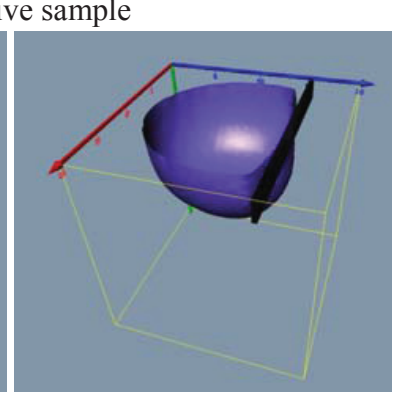

$[10 \mu \mathrm{s}]$

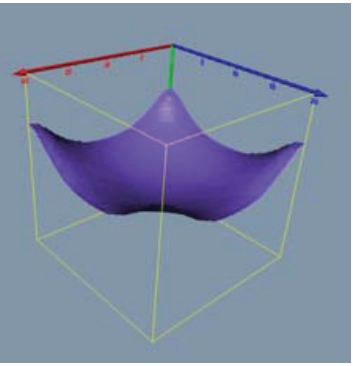

$[15 \mu \mathrm{s}]$

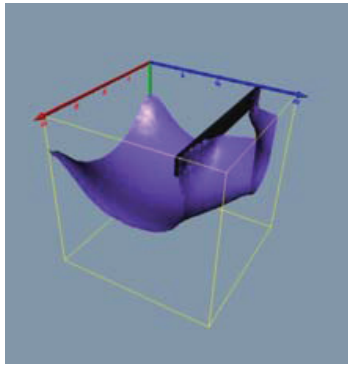

$[15 \mu \mathrm{s}]$

FIGURE 2. The results of mathematical modeling of elastic waves by defect-free and defective samples. The results were visualized in 3D mode using the Voxler 


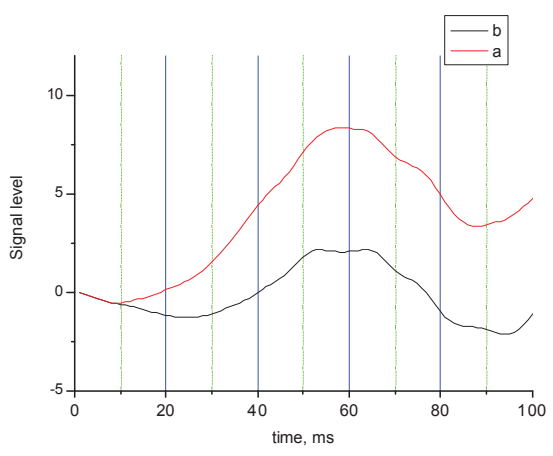

FIGURE 3. Calculated electrical signals from the defect-free (a) and defective (b) samples

The size of the sample was $100 \times 100 \times 100 \mathrm{~mm}^{3}$. An artificial defect of $100 \mathrm{~mm}$ in length and $10 \mathrm{~mm}$ in depth was created with a cutoff machine; the defect was in parallel to the sample lengthwise, at the distance of $25 \mathrm{~mm}$ from the lateral surface. The experimental studies were done with the help of a laboratory hardware-software suite, allowing for the production of pulse mechanical excitement of samples and the registration of electrical responses. The measurement technique of the electrical response to shock excitement is described in more detail in the following article [12].

Pulse excitation was applied to the center of the sample. The electric receiver was situated at the distance of $20 \mathrm{~mm}$ from the impact location. The calculation was performed for those conditions.

The following values of properties of the concrete-sand sample were set for the purpose of the calculation: the density $2.3 \times 10^{3} \mathrm{~kg} / \mathrm{m}^{3}$; the elasticity module $3.3 \times 10^{10} \mathrm{~N} / \mathrm{m}^{2}$; the Poisson ratio 0.2 ; the speed of wave propagation $3.6 \times 10^{3} \mathrm{~m} / \mathrm{s}$. To improve the accuracy and convergence, the dimensions of the elements in the finite-element model was $2.5 \times 2.5 \mathrm{~mm}$. The calculation yielded 1024 points captured at a sample rate interval of $1 \mathrm{~ms}$. The 3D finiteelement model comprised 1600 elements. The developed model allows not only for simulating the wave process, but also for obtaining electrical response. Figure 3 shows the electrical response of the defect-free and defective samples.

As seen from the Figure 3 the presence of the defect in the sample leads to the distortion of the original signal at the moment when the longitudinal wave reaches the defect.

Previously conducted experimental studies [9] showed that the presence of defects in concrete leads to a change in the amplitude-frequency characteristics of the electrical response.

Figure 4 shows the frequency-response characteristics of the calculated and experimental electrical response of the defect-free sample. As can be seen in both cases, the presence of a dominant peak at the frequency of about $16 \mathrm{kHz}$ is observed in the signals' spectra. As shown by Y. Lin, M. Sansalone [13] when testing concrete samples of cubic shape, the dominant spectral peak is associated with the velocity of the P-wave in the infinite medium $\left(C_{p}\right)$ and the ratio of cross-sectional dimension $(D)$ :

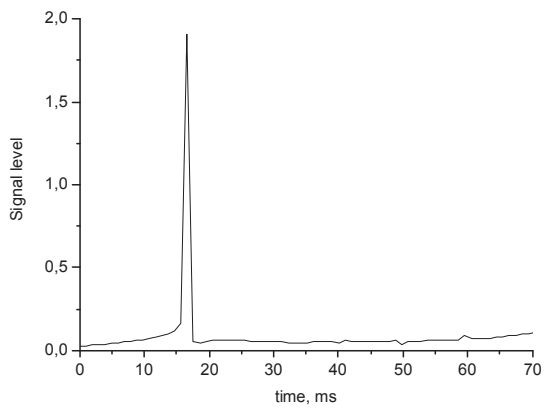

(a)

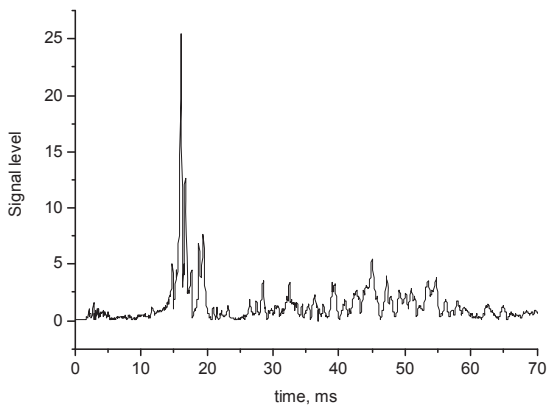

(b)

FIGURE 4. Frequency responses of the calculated model (a) and measured (b) electrical signal 


$$
f_{q}=\frac{0.87 C_{p}}{2 D} .
$$

In our case, the measured value of the velocity of longitudinal waves $C_{p}=3.6 \times 10^{3} \mathrm{~m} / \mathrm{s}, D=0.1 \mathrm{~m}$. Then $f_{d}=$ $15.7 \mathrm{kHz}$ which coincides with the frequency of the main peak both in the calculated and the experimental results. The tests of concrete samples of cubic shape [13] show that the frequency of the dominant spectral maximum $f_{d}$ is related to the P-wave velocity in the infinite medium $\left(C_{p}\right)$ and the cross-section size $(D)$ via the following equation:

$$
f_{d}=\frac{0.87 C_{p}}{2 D} \text {. }
$$

In our case the measured velocity of the P-waves is $C_{p}=3.6 \times 10^{3} \mathrm{~m} / \mathrm{s}$ and $D=100 \mathrm{~mm}$. Thus, the calculated value of $f_{d}$ is $15.7 \mathrm{kHz}$ which precisely corresponds to the calculated and measured waveform signals.

\section{SUMMARY}

The goal of this work was the theoretical and experimental studies of the defects' influence on the electrical response. Based on the results of the three-dimensional simulation of the wave processes formed in the sample at shock excitation, the fields of longitudinal displacements have been obtained. The results of 3D imaging explicitly show the accuracy of the calculations.

The electrical response calculated using the three-dimensional modeling conforms with the experimental results very well. This proves that this modeling is promising for non-destructive testing tasks. On the basis of the results obtained, one can develop additional new opportunities of nondestructive testing of defects by creating a calculated data bank for the comparative analysis and solution of inverse problems.

\section{ACKNOWLEDGEMENT}

This work was carried out within the framework of the "Nauka" state program. The work is performed under the "Nauka" state program, contract No. 13411.1006899.11.065 as of 04.12.2013 with the Russian Ministry of Industry and Trade, the "Polymer" R\&D identifier, RFBR grant 12-01-00069-a, the "Development of a two-step computeraided design of filled polymer systems" project.

\section{REFERENCES}

1. N. J. Carino, in ACI SP-144 Concrete Technology—Past, Present and Future, edited by P. K. Mehta (ACI, Detroit, MI, 1994), p. 632.

2. H. Y. Qasrawi, and I. A. Marie, Cement Concrete Res. 33, 2017 (2003).

3. T. Gudra and B. Stawiski, Ndt. E Int. 33, 1 (2000).

4. D. Algernon, B. Gräfe, F. Mielentz, B. Köhler, and F. Schubert, J. Nondestruct. Eval. 27, 83 (2009).

5. M. Chekroun, L. Le, O. Marrec, O. Abraham, and G. Durand, Ultrasonics 49(8), 743 (2009).

6. Sung Woo Shin, Adeel Riaz Qureshi, Jae-Yong Lee, and Chung Bang Yun, Smart Mater. Struct. 17(5), 17055002 (2008).

7. A. P. Surzhikov and T. V. Fursa, "Mechanoelectrical transformations upon the elastic impact excitation of composite dielectric material", Tech. Phys. (2008).

8. T. V. Fursa and D. D. Dann, Tech. Phys. 56, 1112-7 (2011).

9. T. V. Fursa, K. Yu. Osipov, and D. D. Dann, Tech. Phys. Lett. 37(4), 291 (2011).

10. T. V. Fursa, B. A. Lyukshin, and G. E. Utsyn, Tech. Phys. 58, 263 (2013).

11. R. W. MacCormack, "The effect of viscosity in hypervelocity impact cratering", (1969), p. 69-354.

12. T. V. Fursa, K. Yu. Osipov, B. A. Lyukshin, and G. E. Utsyn, Meas. Sci. Technol. (2014).

13. Y. Lin and M. Sansalone, J. Acoust. Soc. Am. 91, 2674 (1992). 